\title{
Simplification of application operations using cloud and DevOps
}

Anshu Premchand, M. Sandhya, Sharmila Sankar

B. S. Abdur Rahman Crescent Institute of Science and Technology,

GST Road, Vandalur, Chennai 600 048, Tamilnadu. India

\section{Article Info \\ Article history: \\ Received Aug 23, 2018 \\ Revised Oct 27, 2018 \\ Accepted Nov 10, 2018}

\section{Keywords:}

Agility

Application support

Cloud adoption

DevOps

Simplification

\begin{abstract}
In our experience of having worked with financial institutions for over 19 calendar years, we have seen that application development, maintenance and operations teams have a very silo-ed existence. In today's ever more fluid market, it is imperative for these teams to be very agile in their response to business needs. While development teams are trying to adopt agile and devops practices, operations and maintenance teams are sometimes forgotten in this transformation journey even though they play a very crucial role in managing customer facing systems. In this paper, we focus on application operations for their simplification, outline the post production challenges faced by the operations teams, assistance required from the development teams and need for coordination and harmony between application development and operations teams. We use the example of a financial organization where we are currently helping in simplification of application support processes using two key levers of cloud and devops adoption to highlight the path to simplification. We outline the solution levers we used, solution tenets, solution approach and considerations. We also share a target state model and regulated key functions and activities within the model as well as an interim operating model and a target operating model.
\end{abstract}

Copyright $@ 2019$ Institute of Advanced Engineering and Science. All rights reserved.

\section{Corresponding Author:}

Anshu Premchand

B. S. Abdur Rahman Crescent Institute of Science and Technology GST Road,

Vandalur, Chennai 600 048, Tamilnadu, India.

Email: anshuprem@gmail.com

\section{INTRODUCTION}

Software service providers and enterprise development teams are increasingly under pressure to create, enhance and deliver applications at an ever-faster pace. Agile software development practices and devOps help reduce release cycle times but costly and time consuming on-production application errors or mistakes still ail developers, systems administrators and IT support teams irrespective of devOps teams having been established on the application development side. Application problems commonly arise from change of status quo that results in disharmony with upstream and/or downstream systems. The rapid changes caused by agile development therefore necessitate solving application support problems very quickly. Also, the concept of bring your own device to office requires agility to handle application support systems [1]. The existing inter-device collaboration system is generally centralized system [2].

One of primary goals of devOps is to get developers more involved in application maintenance and operations so they can more effectively resolve application issues in production environments [3]. The other important goal is to bring development and testing environments as close to each other as possible. This helps because normally, there are many production issues that cannot be replicated in development environments because they are so disparate. But if the environments are same or similar, the number of 'new' issues occurring in production environments can be brought down measurably. Also, if developers do not have access to live production applications and servers, troubleshooting issues can become very time consuming. 
This leads to a backlog of system defects, unhappy customers, and business and overwhelmed development and operations teams [4]. With increased adoption of big data, Internet of Things and sensor technology by various organization for provisioning smart intelligent services for various application uses, the system becomes complex [5]. In certain industries like financial institutions, roles \& responsibilities dictate access to environments. These are essential for risk \& compliance, amongst other things [6]. We worked with a large financial institution from the fortune 500 list and helped simplify their application support by agility and devOps adoption and delivered quantified benefits between 10-25\% of cost to manage applications for various technology sets. The lower end of the spectrum saw legacy applications and COTS (common off the shelf) product sets and the higher end saw applications written in technologies such as java, Net and newer digital technologies.

In our case with financial institution we mentioned, we used the levers of agility, devops and cloud [7]. The delivery managers at the financial organizations had two primary challenges - one, finding skilled developers to fill open positions to improve output from the team and two, improving throughput and productivity of teams. Developers normally spend a large percentage of their time working on application defects and support problems when their time would be better spent on creating new solutions to further the cause of business. Using devOps and agile practices, we helped improve development processes, added tools and created CI-CD pipelines (continuous integration - continuous delivery) for the financial institution and recorded an overall productivity improvement of $6 \%-15 \%$ while maintaining existing headcounts. This additional capacity was utilized to deliver new solutions to business. In this paper, we share our experience of working with the financial institution on improving \& simplifying their application support processes. With this example, we explain how common barriers to production application support agility can be overcome in a time and cost efficient manner with a DevOps approach supported by agile practice adoption, while reducing the cross-functional friction that commonly arises when deployed applications stop running or show defects [8].

We used a two pronged approach to solve this problem - one, we used devops principles and second, we used cloud (private cloud and public cloud).

\section{THE PROPOSED PROCEDURE: SIMPLIFYING AND IMPROVING APPLICATION SUPPORT IN PRODUCTION}

We observed at the financial institution that most development teams and individual developers spent approximately 20 to 30 percent of their time on application support and defect management. Ideally, the development teams can be granted read-only and audited access to infrastructure resources so they can get the information they need without exposing the organization to unnecessary risks including (and not limited to) financial risks. We helped simplify the operations processes to:

a) Provide cross-functional devOps visibility

b) Maintain security, risk management and compliance policies

c) Control access rights as needed by role

d) Filter, mask and harness sensitive data

e) Audit developer access to production systems

f) Create dashboards for application health monitoring

g) Generating reports as needed and keeping right stakeholders in loop

In our example, with simplified processes, development and operations teams were able to report increase in collaboration and were able to gain shared visibility to solve production application support problems faster and reported lesser defects in production over a period of time. We were able to report increased productivity and improved collaboration throughout their development and operations teams by breaking down silos and creating a unified view of the enterprise. We will explain the method applied in sections that follow. After first and second wave of simplification, we were able to:

a) Reduce operations and support overhead and costs

b) Reduce licensing costs

c) Resolve customer-impacting production issues faster

d) Improve up-time

e) Benefit from greater collaboration and knowledge sharing

f) Get better real-time visibility across various environments and rationalize the number of environments

g) Successfully manage risk, monitoring and audit requirements through role-based access for developers and tracking of all troubleshooting and change activities

h) Easier migration of applications to cloud (as needed)

i) Mitigate \& manage risks and improve effectiveness of outsourced development and support resources

j) Scale on demand the development and operations teams by cross training 
The "Scaling on Demand" worked in two ways:

1. Developers were able to get a dashboard view of all of their applications and related servers. They were able to track the health of the entire application stack. They could, without assistance :

a) Automatically discover, monitor and restart applications

b) Centrally view apps from a data center(s)

c) Manage cloud based applications (if any)

d) Track changes

e) See applications, services and scheduled tasks

f) Access log and configuration files

g) Query production databases

h) Monitor application and server health

i) Get notifications and alerts

2. Operations teams were able to simplify their workload by:

a) Giving development teams safe and secure access to production applications and production servers.

b) Provide cross-functional devOps visibility

c) Maintain security, risk management and compliance policies

d) Control access rights

e) Audit developer access to production systems

We carefully went through all the requirements of the financial organization and applied a multipronged approach. We split the requirements in to two service bundles

a) Bundle one: Traditional IT and Private Cloud

b) Bundle two: Public Cloud

The key motivator was to ensure a flawless digital customer journey and the IT-infrastructure needed to support this. Modernization, streamlining and consolidation were the key themes in structural change at the financial institution we were helping. The financial organization need to drive changes from a business perspective \& for IT to be successful, a strong cooperation and collaboration with the business was necessary. To achieve all these, we proposed adoption of agile and devops practices [9-10]. Table 1 broadly lists our recommendations for the financial organization's business objectives shared with us.

Table 1. Mapping of Business Objectives to Our Recommendations

\begin{tabular}{|c|c|}
\hline Business Objectives & Our Recommendations \\
\hline System, service and business availability needs (24/7) & Highly resilient infrastructure in all layers on cloud infrastructure \\
\hline Capacity fluctuation management & Public cloud for reliability and scalability \\
\hline Simplified tools \& services to manage cost & Simplified, orchestrated tool chain \\
\hline Reduce size of code base & $\begin{array}{c}\text { Consider cloud brokerage service model, cognitive automation for } \\
\text { operational efficiencies }\end{array}$ \\
\hline Simplified integration management & Template based integration \\
\hline $\begin{array}{l}\text { Seamless digital customer journey supported by IT for } \\
\text { improved customer satisfaction }\end{array}$ & Leverage cloud to provide diverse set of IaaS and PaaS services \\
\hline
\end{tabular}

Adopting to the three key themes of structural changes namely Modernization, Streamlining and Consolidation, we constructed the target state solution to meet the current and future requirements of the financial organization.

Figure 1 shows the key solution drivers for the entire exercise. The key drivers were divided into 6 classes which drove one or more key tenets of the solution. These driver classes were cloud, automation, digital platforms, governance, security and cost. For each driver class there were sub-focus areas. For example, for digital, the sub focus areas included cloud bursting, modern integration services, and microservice enablement. For governance, the sub-focus areas were cloud service brokerage, cloud governance, architecture board and competence management for regulations and so on. These drivers helped us arrive that the key tenets of the entire proposed solution for simplification of application operations as we have described below. The key tenets of the proposed solution were:

a) Zero disturbance knowledge transition

b) Cloud assessment enabled with application centric assessment approach

c) Cloud focused approach for workload migrations

d) Migration and transformation risks with mitigation

e) Complete scope of services on IT hosting and operations

f) Scalable and Reliable target cloud platform

g) Focused automation for stable infrastructure

h) Centralized monitoring with event correlation across platforms, applications and network 
i) Cloud governance enabled operating model designed with single point of ownership

j) Quality support

k) Support improvements through operational process improvements

1) Run support for cost efficiencies for both hosting and operational services

m) Manage Risks

n) Allow customer controlled personalization on all new digital applications

o) An ideal team mix with all relevant skills and enabled with cloud governance

p) Operational transparency through service management tools and processes

q) Digital and autonomous platform to address current and future requirements

r) Continuous service improvements through analytics, automation and technology and process driven hosting and support enhancements

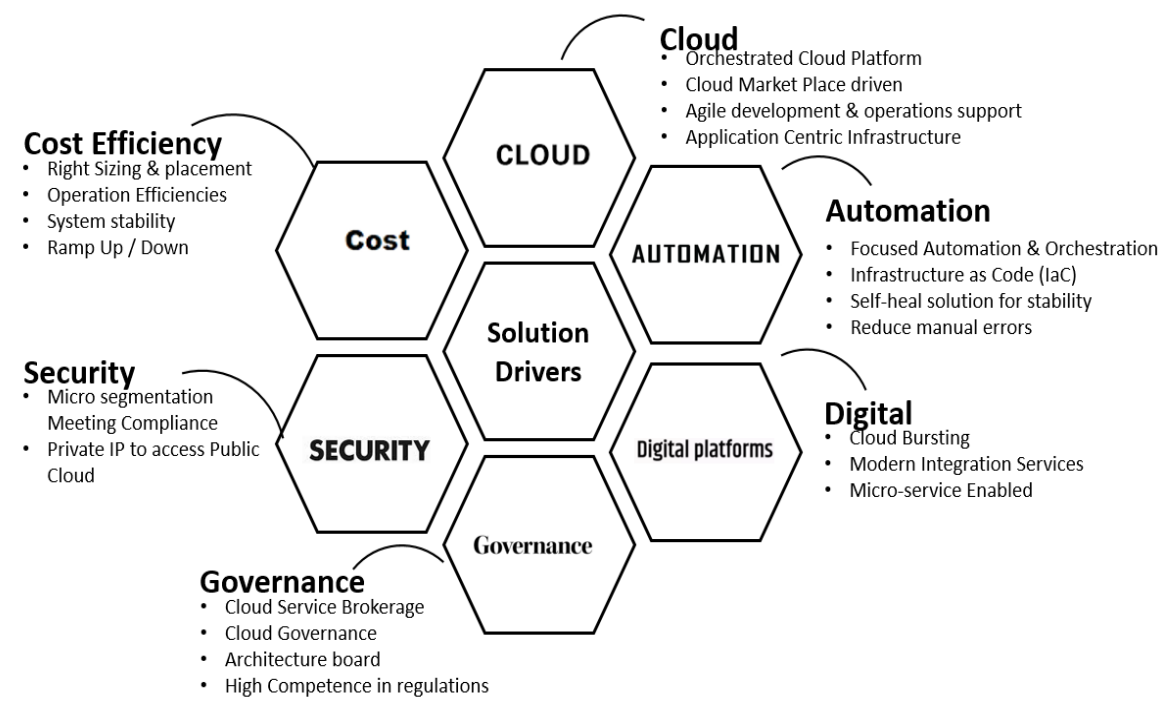

Figure 1. Key solution drivers

Broadly, as Table 2 shows, we classified all the current capabilities and/or services available into these two bundles to clearly specify which one of these services could be handled using traditional IT and private cloud and which ones could be taken to a public cloud solution. Since we were focusing on application support, for this simplification exercise, the following services were deemed out of scope:

a) Application Development - Build, Configure, Test

b) Application Maintenance - 3rd Level Application Support, Minor enhancements, bug fixes

c) Archiving

d) Data Center Decommission

Table 2. Bundle Wise Split of Activities

\begin{tabular}{ccc}
\hline Current Capabilities & Traditional IT \& Private Cloud & Public Cloud \\
\hline Cross Functional Services & Yes & Yes \\
Security Operation Center & Yes & Yes \\
Application Operations & Yes & Yes \\
Integration Service & Yes & Yes \\
Database Management & Yes & Yes \\
Network Services & Yes & No \\
IaaS (Mainframes, legacy) & Yes & No \\
PaaS (all types of databases) & Yes & Yes \\
IaaS (all types of OS) & Yes & Yes \\
Storage Capacity & Yes & No \\
Server Capacity & Yes & No \\
Management of SaaS & No & Yes \\
\hline
\end{tabular}


We also analyzed the existing infrastructure and drew observations and then mapped each of these observations to inferences that helped define the target operating model (TOM) and target simplified application support/operations solution. Some of the important observations and inferences are listed in Table 3.

Table 3. Our Key Observations \& Inferences Drawn

\begin{tabular}{|c|c|}
\hline Observations & Inferences \\
\hline $\begin{array}{l}\text { The existing infrastructure had minimal or no } \\
\text { footprint on public cloud }\end{array}$ & $\begin{array}{l}\text { Consider moving possible non-sensitive workloads to public cloud, } \\
\text { which would enable higher scalability and flexibility in the target } \\
\text { infrastructure }\end{array}$ \\
\hline $20 \%$ of the systems were in physical form & $\begin{array}{l}\text { Possibility of migrating this } 20 \% \text { infrastructure to virtual form which } \\
\text { would give } 100 \% \text { virtual infrastructure, which is an excellent enabler for } \\
\text { digital technologies like Internet of Things (IoT) etc. }\end{array}$ \\
\hline $\begin{array}{l}\text { No backup archival solution even though there were } \\
\text { multiple application based archival solutions } \\
\text { available }\end{array}$ & Possibility of deploying consolidated archival solution with compliance \\
\hline $\begin{array}{l}\text { No cloud management platform and no self-service } \\
\text { portal based provisioning }\end{array}$ & $\begin{array}{l}\text { Deployment of hybrid cloud platform enabled with user friendly } \\
\text { interfaces which help with faster go-to market application deployments }\end{array}$ \\
\hline Multiple versions of operating systems & Operating systems standardization while application migration \\
\hline Legacy integration platforms & Modernization of current integration platforms \\
\hline No automated disaster recovery solution & Possibility of bringing in DR automation \\
\hline
\end{tabular}

\section{THE METHOD}

We also followed data-based approach of performing assessment of the current infrastructure and workloads to qualify the cloud workloads and understand the current architectural gaps. As a part of the solution we proposed to perform a quick assessment for cloud adoption during transition. We wanted to assess, design, build and migrate the infrastructure over the period of 6 to 8 months as per the agreed migration timeline. Our plan was to divide these tasks into 3 distinct phases:

a) Phase 1: We would perform discovery and cloud assessment ( 3 Months) as a part of transition to find out the current infrastructure state and qualify the candidates for migration to cloud (both private and public cloud). The recommended that the assessment report be analyzed by the customer and based on customer's feedback the target state infrastructure will be designed. The target state architecture will get approved by the customer prior to deployment.

b) Phase 2: In phase 2, our plan was to construct the target infrastructure on enterprise cloud infrastructure located in customer's geographic location. This was done to ensure that data related compliance needs were met. Current workloads on incumbent datacenters would be migrated to the cloud in a phased manner over the period of 4 to 6 months (excluding assessment). The target infrastructure was to be built based on the design accepted by customer, all relevant cloud and management tools would be deployed and integrated. Customer application specific service catalogues would be created and deployed on recommended ITSM tool and integrated with cloud management platform.

c) Phase 3: In phase 3, we would be providing end to end cloud services for the financial organization with strong cloud governance in place. We were to be acting as a cloud service brokerage (CSB) provider to ensure that the customer was assured of optimized target infrastructure and ability to adhere to market transformation rapidly.

As shown in Figure 2, we looked at business domain, technology and service delivery as key inputs and mapped these to key transformations such as knowledge management system setup, cloud migration, standardization \& governance, API enabled integration and digital transformation. The figure shows the movement from start to end state with timeline moving from 1-ni weeks with initiatives in the timelines for various key transformations. This helped us draw a business centric migration and transformation approach with timelines where we could target and track each transformation from start to end state. Business Centric approach was applied at all layers of the infrastructure to bring in the complete benefits to the financial organization. Data center was treated as scalable platform which would bring speed and agility in business application deployments, servers were treated as group of business components hence failure of each component was mapped with failure of business functionality and hence the architecture would be realigned based on the business functionality criticality. 


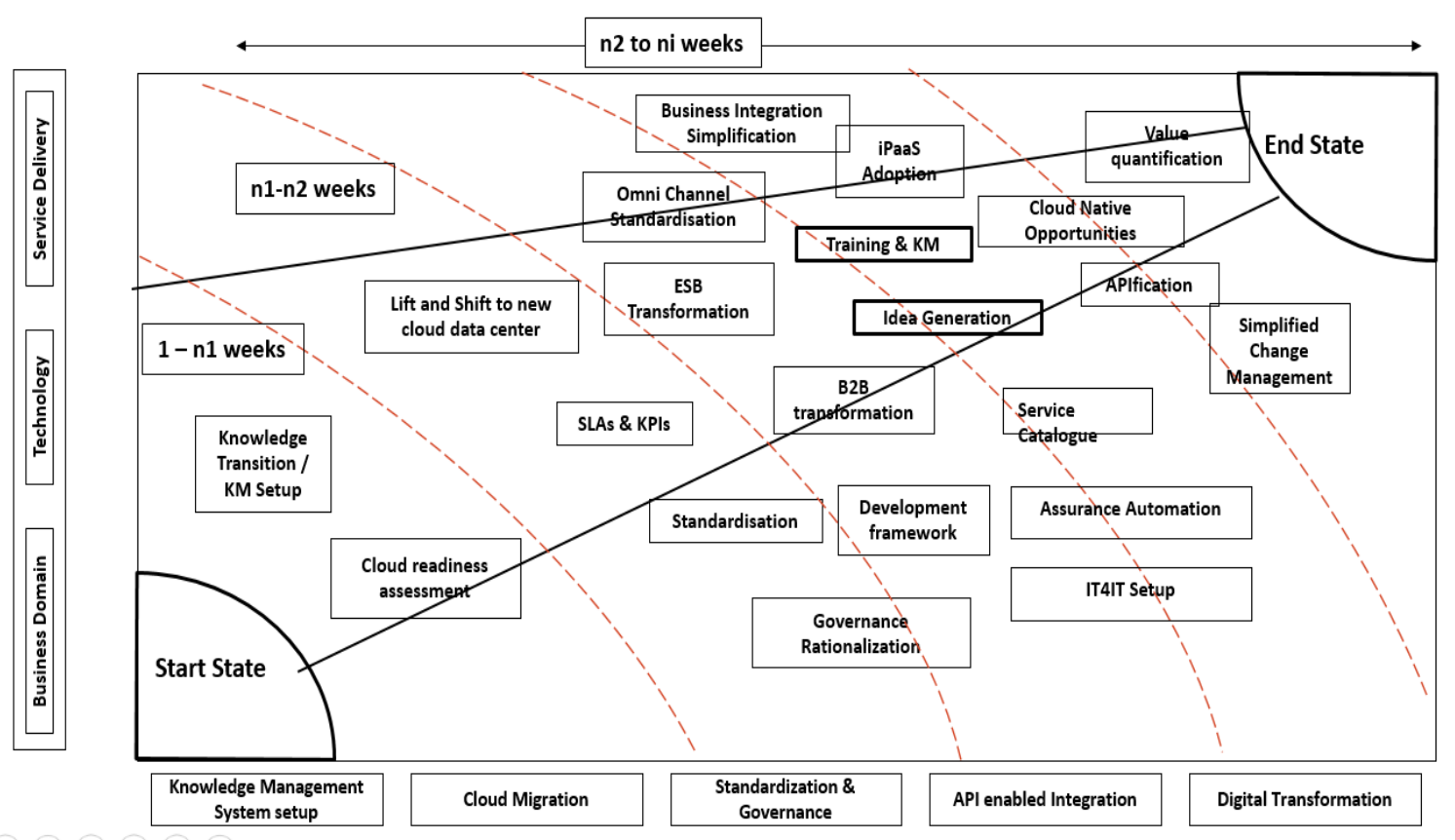

Figure 2. Business centric migration \& transformation approach

\section{SOLUTION CONSIDERATIONS}

The following were the key solution considerations we had taken into account while constructing the target infrastructure:

a) Two cloud infrastructure locations within the country limits as two availability zones to ensure data related compliance requirements are met. One of the locations to also act as primary disaster recovery location

b) We recommended a serious look at "as a service" models to the financial organization to bring down costs, which included IaaS and PaaS services

c) We requested considering to host $50 \%$ split of workloads in between two locations to provide active-active configuration of the workloads

d) Storage tiers and sizing had been considered based on current data provided by the financial organization, to be validated during due diligence stage

e) Backup considerations were based on current requirements given by the customer, this would be validated during due diligence

f) Archival solution was proposed as optional solution

g) Target infrastructure will be enabled with maximum automation possible

h) We recommended considering self-heal solutions be considered as a part of the target infrastructure

i) Consolidated Integration Services platform solution had been considered

j) Target infrastructure is built to ensure cloud service brokerage model delivery

k) The target infrastructure monitoring and management solution had been constructed to handle hybrid cloud platform and legacy devices

1) Business centric migration move groups would be created during transformation

m) Target infrastructure had features enabled for future technology transformations such as Server-less Computing, Digital, and Internet of Things etc.

n) Cloud LAN hardware and software were sized suitably for server workloads and network components which include:
1) LAN Switching Fabric
2) Perimeter \& Internal Firewall
3) Intrusion Detection and Prevention at important Ingress/ Egress Zones
4) External/DMZ (demilitarized zone) and Internal Load Balancer
5) Remote Client Terminal Connection to access financial organization's resources
6) Site to site VPN (virtual private network)
7) Support functions for IP Addresses, DHCP, DNS 


\section{RESULTS AND DISCUSSION: INFRASTRUCTURE SECURITY, MONITORING AND MANAGEMENT}

We recommended that cyber security be updated and transitioned to SIEM (Security Information \& Event Management) Platform and enterprise vulnerability management, identity access management, public key infrastructure and other platform L1/L2/L3 security services be spruced up in different business verticals. We recommended the following services be delivered as part of security operations center for the financial organization:

a) Security Information and Event Management

b) Vulnerability Assessment

c) Penetration Testing

d) Identity and Access Management

e) Public Key Infrastructure

We understood that the financial organization's long term strategy was to move enterprise workloads to public cloud. Therefore, our recommendations were the following:

a) Unified infrastructure and application monitoring

b) Continuous discovery and automated relationship maps

c) Financial analytics on public cloud expenses, cost and resource optimizations

d) Unified dashboards \& reports for cloud operations

We will detail the security, monitoring and management solution in our next paper.

Application Operations Rationalization using DevOps for Simplified Management:

We recommended that the financial organization strategically move towards a devOps model for their application operations, maintenance and development work. The main driver for this change was a need to improve time-to-market for new application functionality or other code changes.

We proposed DevOps as a Service (DaaS) model which povides all the benefits of devOps as a managed cloud service. Based on the DevOps Maturity Assessment of the financial organization's current state, we will be able to create a time-bound, milestone based plan to automate as much as possible and bring the entire continuous deployment pipeline (CD) onto the public cloud. This would enable the financial organization to focus on other areas without having to worry about the devOps stack management and IT4IT work that will come under the "Managed DaaS" umbrella.

During Due Diligence phase, a feasibility analysis would be done jointly with the financial organization to understand all of their specific needs and provide customized solutions leveraging public cloud for devOps adoption keeping in mind various other factors like cost, performance of tools and orchestration needs. From the state of technology operations of the financial organization, we understood that one-pass move to the target state we wanted to reach would be impossible. We would have to target to reach an intermediate operating model where all simplification themes were underway. This would also help us keep track of progress and course correct as needed.

As collaboration is key for a successful devOps model, we recommended that Application Operations (AO) team will perform the following activities:

a) Provide clear definition of processes and responsibilities across the devOps organizations (lines of business within the financial organization) and the application operations organization

b) Roadmap for the implementation covering all relevant areas such as suggesting a stepwise approach

c) With the sequence of implementation and pace for roll-out - such as area to start with and how to gradually scale up

d) Selection and implementation of tools supporting devOps

e) Actual implementation

f) Orchestration across the tool-chain

Figure 3 shows the target operating model for the application support services of the financial organization. We are on track to attain the same. The vertical units refer to shared functions across the organization like audit and regulatory, IT4IT teams, risk and compliance management etc. The horizontal units on top are the business groups, business users and service desk that consume the services below them. Cloud management platform works on top of the business aware command center which automation layer that works on top of the other platforms such as legacy platforms, application operations and so on. The final horizontal layer represents the enterprise cloud and public cloud service providers. 


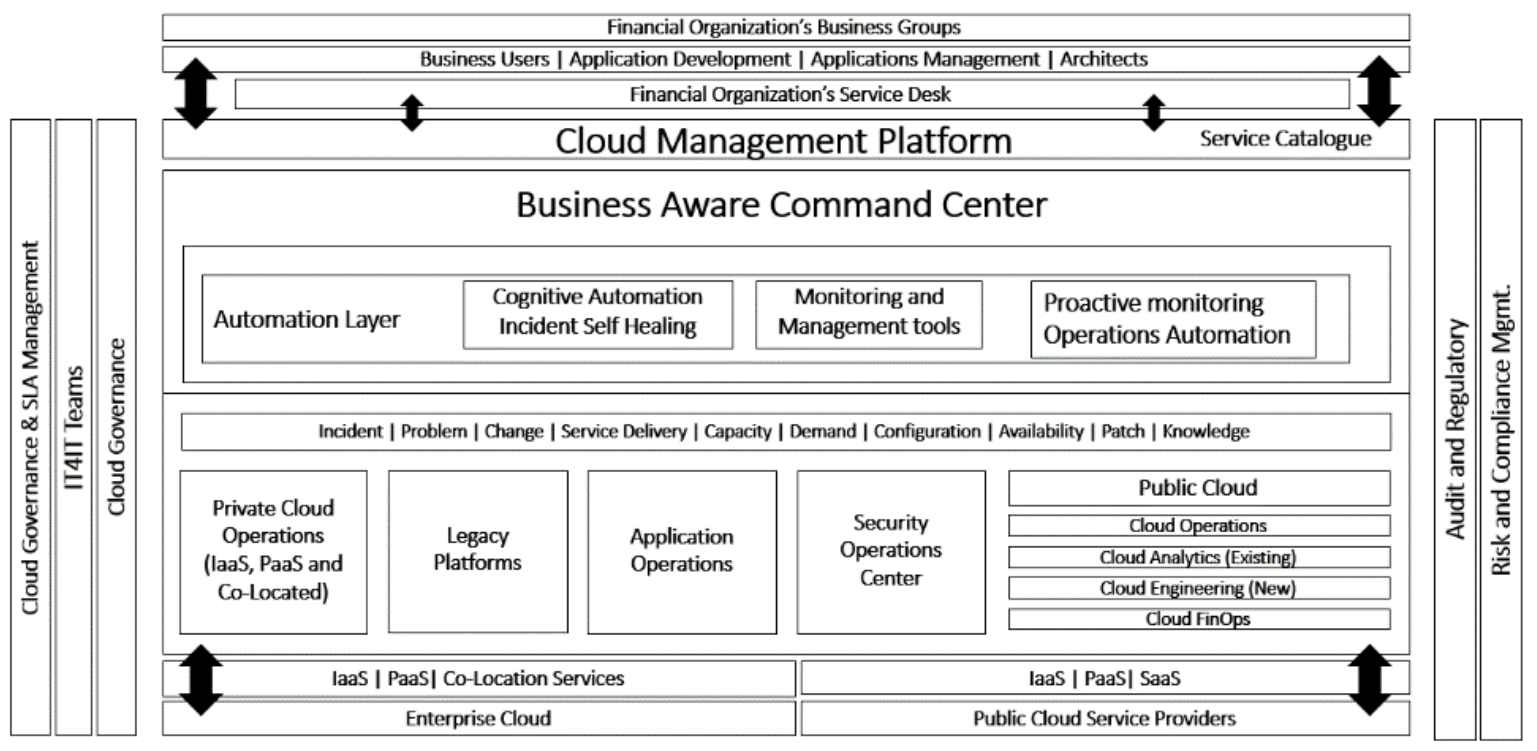

Figure 3. Target operating model

We also requested support from the financial organization and its application development \& maintenance (AD/AM) teams. The following dependencies on the application development $\&$ maintenance $(\mathrm{AD} / \mathrm{AM})$ teams was foreseen:

a) The selection of tools (e.g., deployment tools) shall be aligned with the financial organization's initiatives for automated deployment on test and production.

b) Definition of when different applications/teams are ready for transformation

c) Availability (time and focus) of development team to absorb knowledge and practice the new capabilities

d) Dependency on other application development teams for release coordination

\section{CONCLUSION}

Simplification using cloud and devops adoption cannot happen until operations and infrastructure organizations are made a part of the simplification process and rationalized. These groups need to be disrupted and transformed into an effective devops model to ensure business needs of today's digital world are met. Agile \& devops practices in culture, automation, lean, measurement and collaboration aspects are great enablers for simplification of operations and infrastructure organizations and cloud adoption (private/ public/hybrid) is an excellent enabler of the simplification itself. These levers lead to quantifiable benefits. In a short while, we will be able to write another paper to share the quantified benefits accrued to the financial organization referred in this paper due to the simplification of application support services as we have outlined here. Operations and infrastructure teams may also need to re-skill, cross skill and align to the new way of working and also use cohesive business centric key process indicators. The post production challenges and assistance needed from development teams we have discussed will find resonance in many similar organizations. The solution tenets, solution approach, interim and target operating model can act as a guiding force for organizations with similar issues. Through the transformational approach we have outlined in this paper the application operations teams will be in the driver's seat in the organization's journey towards simplification and delivering greater business value to all stakeholders.

\section{REFERENCES}

[1] Mohd Yusri Jusoh, Haryani Haron, Jasber Kaur, "Work Process of Bring Your Own Device to Support Green Computing", Indonesian Journal of Electrical Engineering and Computer Science;2018; Volume 11, No 1: 137-143.

[2] Changsu Kim,Hankil Kim, Jongwon Lee, Hoekyung Jung, "Web Server-based Distributed Machine Socialization System" International Journal of Electrical and Computer Engineering; 2018; Volume 8, No.2: 631-637.

[3] Michael Fauscette, Randy Perry, Simplifying IT to Drive Better Business Outcomes and Improved ROI", Introducing the IT Complexity Index; IDC; 2014;1-3. 
[4] Anshu Premchand, Dr. Sandhya M, Dr. Sharmila Sankar, "Roadmap for simplification of enterprise architecture at financial institutions", 2016 International Conference on Computation of Power, Energy Information and Communication (ICCPEIC); 2016; IEEE-2016: 511 - 513.

[5] Heena kousar, B.R. Prasad Babu, "Multi-Agent based MapReduce Model for Efficient Utilization of System Resources", Indonesian Journal of Electrical Engineering and Computer Science(IJEECS); 2016; Volume 11, No. 2: 504-514.

[6] Alan Siegel, Irene Etzkorn” Simple: Conquering the Crisis of Complexity” Twelve; 2013: 12-15.

[7] D. Cohen, M. Lindvall, and P. Costa, "Advances in Computers, Advances in Software Engineering, chapter An Introduction to Agile Methods; Elsevier, Amsterdam; 2004: 3-5.

[8] T. Dybå, T. Dingsøyr, "Empirical studies of agile software development: A systematic review", Information \& Software Technology; 2008; 50(9-10):833-859.

[9] S. Ilieva, P. Ivanov, and E. Stefanova, "Analyses of an agile methodology implementation", Proc. of the 30th EUROMICRO Conference (EUROMICRO 2004); 2004: 326-333.

[10] D. Karlstr"om, P. Runeson, "Combining agile methods with stage-gate project management", IEEE Software; 2005; 22(3): 43-49. 